\title{
BK ablation attenuates osteoblast bone formation via integrin pathway
}

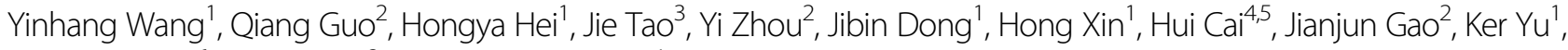
Svetlana Reilly, ${ }^{6}$ Peihao Yin ${ }^{3}$ and Xuemei Zhang ${ }^{1}$

\begin{abstract}
Impaired bone formation is one of the major causes of low bone mass and skeletal fragility that occurs in osteoporosis. However, the mechanisms underlying the defects in bone formation are not well understood. Here, we report that big conductance calcium-activated potassium channels (BKs) are required for bone formation and osteoblast function both in vivo and in vitro. By 15 weeks of age, BK knockout (BKO) mice exhibited a decline in bone mineral density and trabecular bone volume of the tibiae and lumbar vertebrae, which were associated with impaired bone formation and osteoblast activity. Mechanistically, BK ablation in bone and bone marrow mesenchymal stem cells (BMSCs) of BKO mice inhibited integrin signaling. Furthermore, the binding of a subunit of BK with integrin $\beta 1$ protein in osteoblasts was confirmed, and FAK-ERK1/2 signaling was proved to be involved by genetic modification of KCNMA1 (which encodes the a subunit of BK) in ROS17/2.8 osteoblast cells. These findings indicated that BK regulates bone formation by promoting osteoblast differentiation via integrin pathway, which provided novel insight into ion transporter crosstalk with the extracellular matrix in osteoblast regulation and revealed a new potential strategy for intervention in correcting bone formation defects.
\end{abstract}

\section{Introduction}

Defects in bone formation in response to the accelerated bone resorption cause an imbalance in bone remodeling resulting in low bone mass and skeletal fragility in osteoporosis $^{1,2}$. These defects are characterized by a decrease in cell number and function of osteoblasts that are thought to be related to a decline in growth factors (e.g., insulin-like growth factor-1, IGF-1), a deficiency of estrogen and androgen, an accumulation of tumor necrosis factor alpha (TNF $\alpha$ ) or ageing; ${ }^{3-5}$ the detailed mechanisms underlying changes in osteoblast functional responses are not understood.

\footnotetext{
Correspondence: Xuemei Zhang (xuemzhang@fudan.edu.cn)

'Department of Pharmacology, School of Pharmacy, Fudan University, Shanghai 201203, China

${ }^{2}$ Department of Bone Metabolism, Institute of Radiation Medicine, Fudan University, Shanghai 200032, China

Full list of author information is available at the end of the article.

These authors contributed equally: Yinhang Wang, Qiang Guo, Hongya Hei

Edited by A. Stephanou
}

Recently, one of the subtypes of potassium $\left(\mathrm{K}^{+}\right)$channels, the big conductance calcium-activated potassium channel (BK, also named Maxi K or KCa1.1), has gained considerable interest in bone remodeling ${ }^{6}$, as global deletion of BK channels in mice caused osteopenia due to enhanced osteoclast resorption secondary to the autonomous release of cathepsin $\mathrm{K}$ (one of the major enzymes in the degradation of bone matrix $)^{6}$. Potassium channels are a diverse family of membrane proteins that include calcium $\left(\mathrm{Ca}^{2+}\right)$-activated potassium channels (termed $\mathrm{Kca}$ ), voltage-gated potassium $\left(\mathrm{K}^{+}\right)$channels (termed $\mathrm{Kv}$ ) and others (K2p and Kir channels) ${ }^{7,8}$. It has been shown that the activation of Kca channels increases the efflux of $\mathrm{K}^{+}$ and limits $\mathrm{Ca}^{2+}$ influx coherently through the deactivation of voltage-gated $\mathrm{Ca}^{2+}$ channels, which are suggested to be involved in cell signal transduction and viability in excitable cells, such as neurons, cardiomyocytes and smooth muscle cells and in the regulation of $\mathrm{K}^{+}$homeostasis or cell volume in non-excitable cells, such as tumor cells, bone cells and kidney epithelium ${ }^{9}$. The Kca

\section{(c) The Author(s) 2019}

(c) (i) Open Access This article is licensed under a Creative Commons Attribution 4.0 International License, which permits use, sharing, adaptation, distribution and reproduction cc) in any medium or format, as long as you give appropriate credit to the original author(s) and the source, provide a link to the Creative Commons license, and indicate if changes were made. The images or other third party material in this article are included in the article's Creative Commons license, unless indicated otherwise in a credit line to the material. If material is not included in the article's Creative Commons license and your intended use is not permitted by statutory regulation or exceeds the permitted use, you will need to obtain permission directly from the copyright holder. To view a copy of this license, visit http://creativecommons.org/licenses/by/4.0/. 
channels are subdivided into three types according to their conductance: big conductance Kca (BK) channels, intermediate conductance (IK) channels and small conductance (SK) channels ${ }^{7}$. The BK channel consists of four pore-forming $\alpha$-subunits and several regulatory $\beta$ or $\gamma$ subunits $^{10-12}$. The uniqueness of the channel is that it can be activated by both changes in voltage and intracellular $\mathrm{Ca}^{2+}$ concentration, which link intracellular $\mathrm{Ca}^{2+}$ with membrane electrical signaling ${ }^{10}$. In neurons, heart and bladders, BK channels participate in vascular contractility regulation, neurotransmitter release and hormone secretion ${ }^{10,11}$. Recent reports in non-excitable cells, including bone cells, suggest BK channels regulate cellular functions through intracellular biochemical mechanisms rather than electrical signaling ${ }^{12,13}$.

BK channels are expressed in primary osteoblasts ${ }^{14-16}$, osteoblast cell lines (e.g., MG63, SaOS2, and ROS17/2, $8)^{14,16-21}$ and progenitors (e.g., mesenchymal stem cells $)^{22-24}$. Emerging evidence indicate that the BK channels in osteoblasts may act as mechanosensors to transduce extracellular mechanical signals into cell; Davidson et al. was the first to report activation of BK channels in human G292 osteoblast-like osteosarcoma cells by the membrane stretch; ${ }^{19,25}$ in human ondonosteoblasts, BK channels also exhibit mechanosensitivity ${ }^{26}$.

The focal adhesion kinase (FAK) is an important player in mechanotransduction; recent studies show that the interaction of FAK with the C-terminus of BK channels is markedly enhanced by hypotonic shock-induced cellular deformation in human osteoblasts ${ }^{16,27}$. BK channels were also found in the activation of tyrosine phosphorylated prtein Syk by PGE2. FAK and Syk tyrosine kinase belong to the family of cytosolic tyrosine kinases and involve in cytoskeleton organization and signal transduction mediated by integrins ${ }^{28-31}$. Induction of integrin $\beta 1$ leads to FAK aggregation and phosphorylation (auto-phosphorylation at Y397 site) activates MAPK and ERK kinases, and transcription factors-Runx2 and Osterix ${ }^{32-35}$. Runx2, in turn, triggers a cascade of osteoblast markers, including osteopontin (OPN), collagen I, alkaline phosphatase (ALP) and osteocalcin (OCN), to promote the differentiation of osteoblasts for bone matrix synthesis and mineralization $^{32,35}$.

The BK channels are reported to affect cell proliferation and differentiation; for instance, BK channel blockers, TEA and tetrandrine, cause significant changes MG63 cell numbers; ${ }^{16,27}$ in the same cell type secretion of OCN is modulated by BK ${ }^{17}$. Similarly, silencing BK in human BMSCs decrease osteogenic differentiation by reducing mineral precipitation and $\mathrm{OCN}^{24}$. We have previously observed that BK knockout in ROS17/2.8 osteoblast cells posses a lower expression of runt-related transcription factor 2 (Runx2, the core transcription factor in osteoblast differentiation) associated with a reduction in osteoblast markers $\mathrm{OPN}$ and $\mathrm{OCN}^{21}$. Activity of the BK channels can be regulated by prostaglandin E2 (PGE2) ${ }^{18,25,36}$, via the recruitment and activation of tyrosine phosphorylated protein Syk. These channels can also be activated by parathyroid hormone $(\mathrm{PTH})^{18}$, likely by the triggered increase in intracellular $\mathrm{Ca}^{2+}$ concentration and estrogens ${ }^{37,38}$ that are major modulators of bone remodeling ${ }^{39}$. Moreover, the expression of BK is downregulated in the long bones of the osteoporosis model induced by ovariectomy (Supplemental Fig. 1). These findings suggest that BK channels might play a role in the regulation of bone formation under physiological and pathological conditions.

To test this hypothesis, we generated BK knockout mice using the CRISPR-Cas9 system and examined the bone formation index (indices) both in vivo and in vitro. The expression of KCNMA1 (which encodes the $\alpha$ subunit of BK) was further modified (silenced or overexpressed) in osteoblast cell lines, and the role of the integrin pathway in BK signaling was explored.

\section{Results}

\section{Bone loss in BKO mice}

BK channel knockout mice (BKO) were generated by the deletion of exon 4 of KCNMA1 (which encode the pore-forming $\alpha$-subunits of BK) using the CRISPR/ Cas9 strategy. (Fig. 1a). The BKO female mice, which carried a 532-bp fragment deletion from 23591392 to $23591923 \mathrm{bp}$ in the KCNMA1 genome DNA sequence (NC 000080.6), was identified by PCR (Fig. 1b) and confirmed by sequencing (Fig. 1c) and whole cell patch clamp study (Supplemental Fig. 2).

The significant reduction of trabecular bone in the tibiae and lumbar vertebrae was revealed by microcomputed tomography $(\mu \mathrm{CT})$ analysis in the $\mathrm{BKO}$ female mice at 15 weeks of age (Fig. 1d, g). The trabecular bone volume/tissue volume (Tb. BV/TV) in the tibia of BKO mice was markedly decreased by $56.80 \%$ $(P<0.05)$ compared with that of their wild-type (WT) littermates; the trabecular thickness (Tb.Th) was decreased by $17.83 \%(P<0.05)$, and the trabecular number (Tb.N) was decreased by $47.50 \%(P<0.05)$, whereas trabecular separation (Tb.Sp) was increased by 130.68\% $(P=0.06)$ (Fig. 1e). Additionally, the cortical bone volume (Ct.BV) of the proximal tibiae in BKO mice was decreased by $27.75 \%(P<0.01)$ compared with that of their WT littermates, along with decreases in intersection surface area (Ct.Ar) and thickness (Ct.Th) (Fig. 1f). These differences were greater in the long bone (tibia) than in vertebral (lumbar) bone. The BKO mice showed a significant decrease in the trabecular bone mineral density (Tb.BMD) of the proximal tibia and lumbar vertebrae by $36.6 \%(P<0.05)$ and $17.4 \%(P<$ $0.05)$, respectively (Fig. 1e, h). 


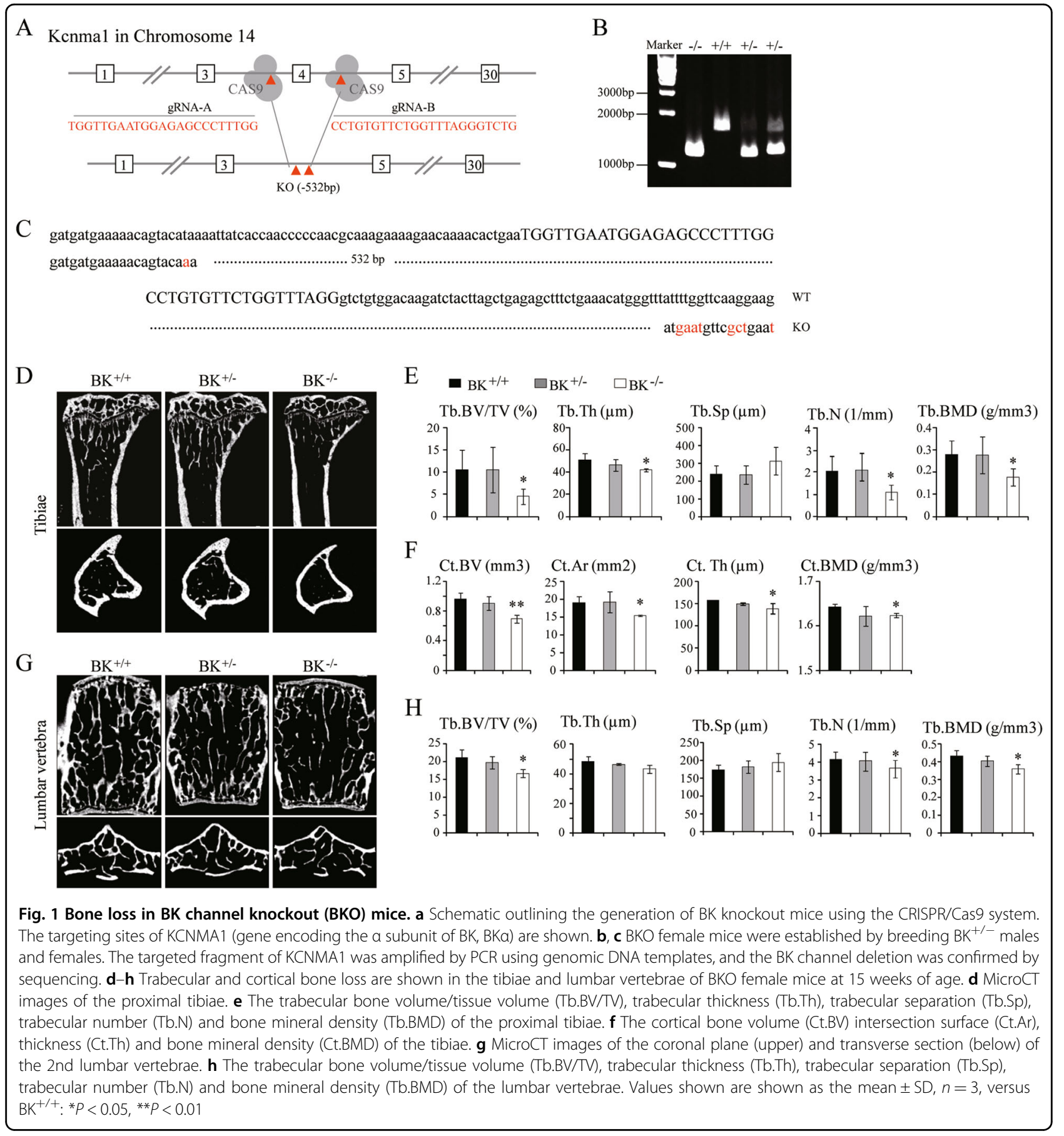

\section{Defects in osteoblast bone formation}

Using histomorphometry, we found that BKO mice have decreased by $31 \%$ osteoblast activity (Fig. 2a, b) shown by the osteoblast perimeter per trabecular perimeter (Ob.Pm/Tb.Pm), compared with that in WT mice; by contrast, there was no significant difference in osteoclast activity, as shown by the osteoclast perimeter per trabecular perimeter (Oc.Pm/Tb.Pm) in Fig. 2c, d. The bone formation rate (BFR/B.S) and the mineral apposition rate (MAR) were decreased by $57.8 \%(P<$ $0.01)$ and $12.5 \%(P<0.01)$, respectively (Fig. $2 \mathrm{e}-\mathrm{g})$. Furthermore, the gene expression levels of Runt-related transcription factor 2 (Runx2) and the osteoblast marker ALP were significantly decreased in the bones of BKO mice as determined by real-time RT-PCR (Fig. 2h). The ratio of the gene expression of receptor activator of nuclear factor kappa B ligand (RANKL), the major activator of osteoclasts, to osteoprotegerin (OPG), which 
A

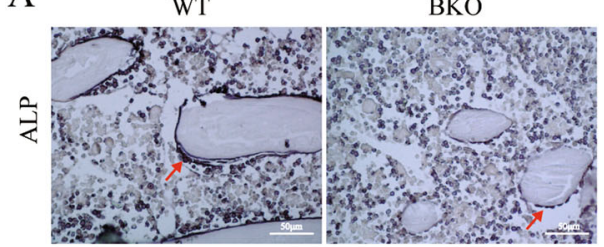

C

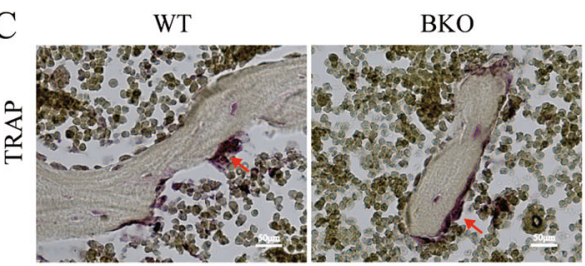

E
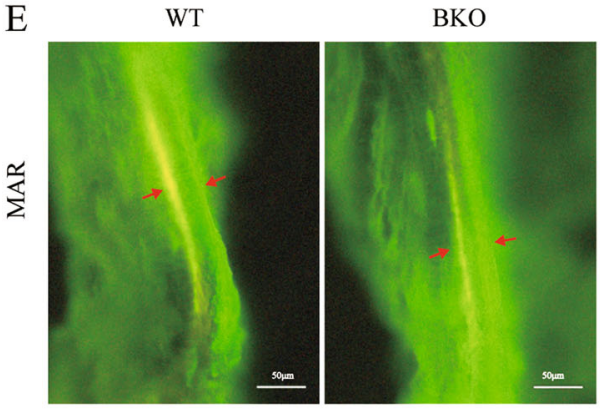

$\mathrm{K}$

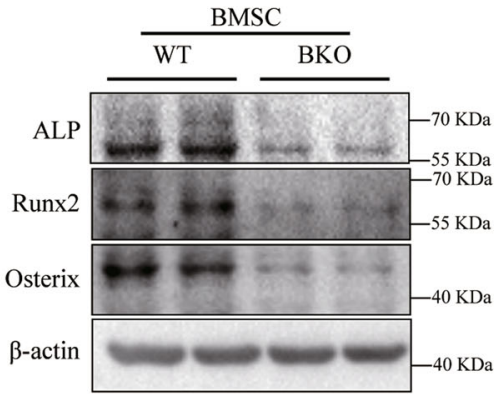

B

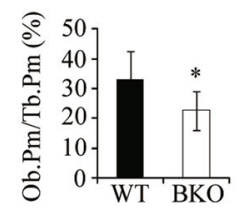

D
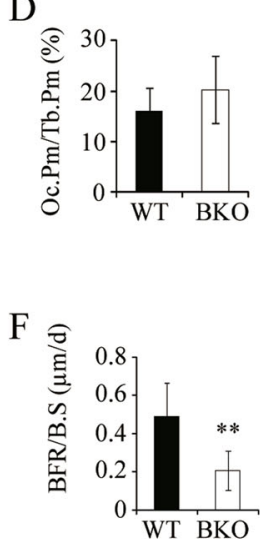

G
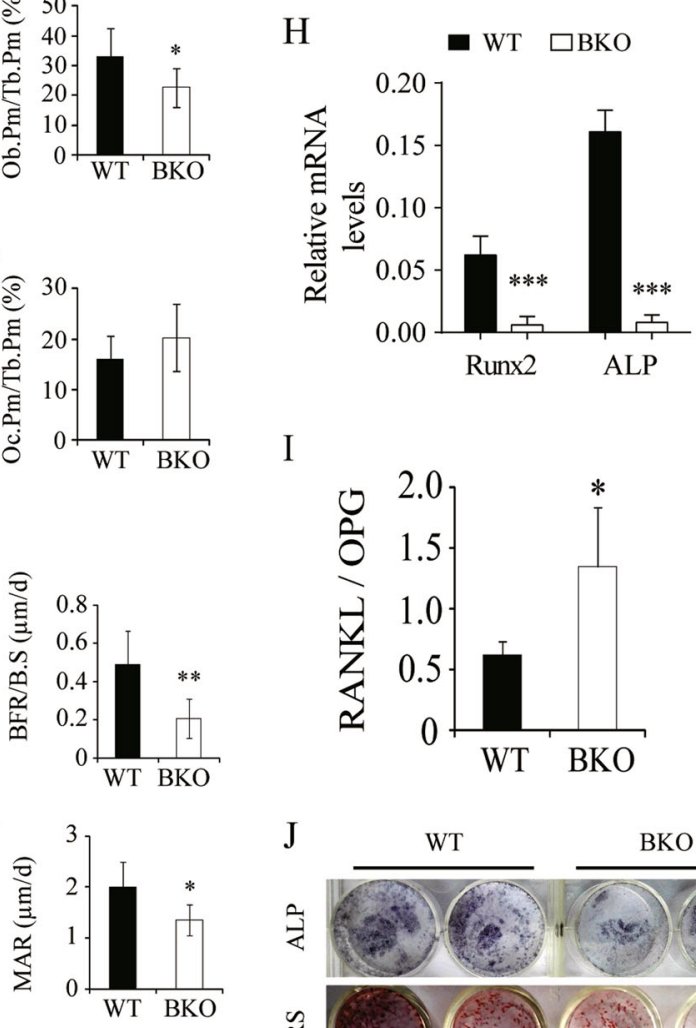

I
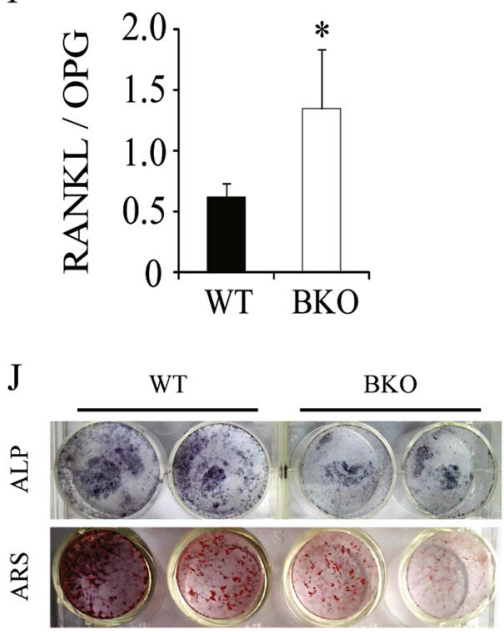

L

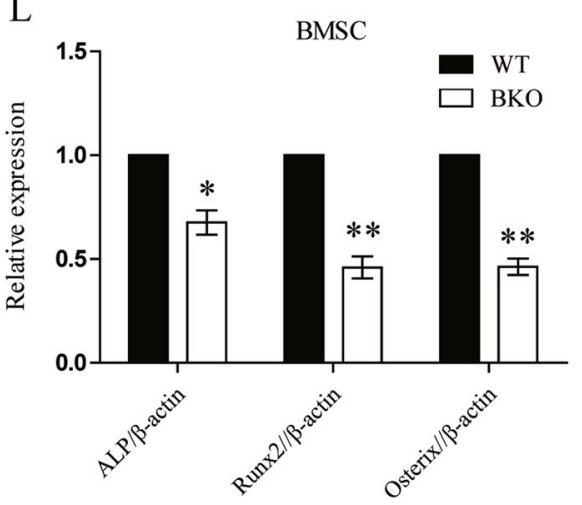

Fig. 2 Defects in osteoblast bone formation in BKO mice. a ALP staining (scale bar $=50 \mu \mathrm{m}$ ) in the trabecular region of the lumbar vertebrae, and the red arrows pointed to the typical osteoblasts. $\mathbf{b}$ The osteoblast perimeter (Ob.Pm/Th.Pm) in the trabecular region of the lumbar vertebrae. $\mathbf{c}$ TRAP staining $($ scale bar $=50 \mu \mathrm{m})$ in the trabecular region of the lumbar vertebrae, and the red arrows pointed to the typical osteoclasts. $\mathbf{d}$ The osteoclast perimeter (Oc.Pm/Th.Pm) in the trabecular region of the lumbar vertebrae. e Tetracycline/calcein labeling (the yellow line is tetracycline labeling and the green line is calcein labeling, scale bar $=50 \mu \mathrm{m}$ ), $\mathbf{f}$ bone formation rate (BFR/B.S) and $\mathbf{g}$ mineral apposition rate (MAR) in the trabecular region of the tibiae. Defects in bone formation are shown in BKO mice. Values are shown as the mean $\pm \mathrm{SD}, n=6$, versus WT: ${ }^{*} P<0.05$, ${ }^{* *} P<0.01$. $\mathbf{h}, \mathbf{i}$ Gene expression of Runx2 and ALP and the ratio of RANKL/OPG were determined in the femurs of BKO and WT mice by real-time RT-PCR. Values are shown as the mean $\pm S D, n=4$, versus WT: ${ }^{*} P<0.01,{ }^{* *} P<0.005$. $\mathbf{j}$ ALP and Alizarin Red staining (ARS) of BMSCs after 14 and 21 days of culture, respectively, in DMEM supplemented with $50 \mathrm{\mu g} / \mathrm{ml}$ ascorbic acid and $10 \mathrm{mM}$ B-glycerophosphate. $\mathbf{k}$, I Expression and quantitative analysis of ALP, Runx2, and osterix of BMSCs after 21 days of culture in DMEM supplemented with $50 \mu \mathrm{g} / \mathrm{ml}$ ascorbic acid and $10 \mathrm{mM}$ B-glycerophosphate. Values are shown as the mean $\pm \mathrm{SD}, n=6$, versus $\mathrm{WT}:{ }^{*} P<0.05,{ }^{* *} P<0.01$ 
is a decoy receptor for RANKL, was modestly increased (Fig. 2i).

The decreases in osteoblast function in BKO mice were further verified by decreases in alkaline phosphatase (ALP) activity and its ability to form mineralized nodules, as determined by ALP and Alizarin Red staining, respectively, in bone marrow stromal cell (BMSC) cultures (Fig. 2j). Consistently, the expression levels of Runx2, Osterix, and ALP were significantly decreased in BK-deficient BMSCs (Fig. 2k, l). Taken together, these data indicate that BK channel ablation inhibits the osteoblast differentiation and bone formation.

\section{FAK/ERK involved in BK action in osteoblasts}

Interestingly, along with the impaired osteoblast differentiation, BK ablation caused a decrease in the phosphorylation of focal adhesion kinase (p-FAK-Y397) and p-ERK1/2 in the bone and BMSCs of BKO mice as determined by western blot analysis (Fig. 3a-d).

To explore the underlying molecular events, the expression of KCNMA1 was further modified in ROS17/ 2.8 osteoblast cells. Three BK $\alpha$-shRNA plasmids Y2255, Y2256, and Y2257 were transfected into ROS17/2.8 cells. As shown in Fig. 3e, f, BKa was knocked down at the protein level by Y2255 plasmid. In line with findings in BKO mice, silencing BK expression caused a significant decrease in Runx2, osterix, and ALP (Fig. 3g, h), along with p-FAK-Y397 and p-ERK1/2 (Fig. 3i, j), which are two major mediators of signal transduction via integrins. Similar results are also found in MC3T3-E1 osteoblasts after BK knocking down (Supplemental Fig. 3a, b).

To further explore the BK actions in osteoblasts, we next overexpressed $\mathrm{BK}$ channels using the $\mathrm{BK} \alpha-\mathrm{myc}$ plasmid in ROS17/2.8 cells (Fig. 4a-d) and MC3T3-E1 cells (Supplemental Fig. 4a-d). As shown in the results, overexpression of BK led to the increased protein level of Runx2 that was blocked by the FAK inhibitor PF-562271 (Fig. 4a, c) or the ERK1/2 inhibitor U0126 (Fig. 4b, d). The increase in Runx2 in the nucleus of osteoblasts was confirmed by immunofluorescent staining (Fig. 4e, f). Similar results were observed in MC3T3-E1 cells (Supplemental Fig. 4a-d). These results indicate that the FAK-ERK1/ 2 signaling pathway is involved in the activation of Runx2 by BK in osteoblasts.

\section{Interaction between $B K$ and integrin $\beta 1$ in osteoblasts}

Genetic deletion of the BK in mice showed reduced levels of integrin $\beta 1$ in bone and BMSCs (Fig. 5a-d). Similar the expression levels of integrin $\beta 1$ were decreased in the BK knockdown ROS17/2.8 (Fig. 5e, f) and MG63 cells (Supplemental Fig. 5a), while BK overexpressing MG63 cells had increased integrin $\beta 1$ (Supplemental Fig. $5 b)$. To test whether integrin $\beta 1$ regulates BK expression, we knockdown integrin $\beta 1$ with the integrin $\beta 1$-shRNA plasmid. The results showed that downregulation of integrin $\beta 1$ did not affect the expression of BK (Supplemental Fig. 5c, d). Thus BK is likely to be located upstream of integrin $\beta 1$ signaling in osteoblasts.

To determine the interaction of BK with integrin $\beta 1$ in osteoblasts, immunocytochemical staining (ICC) and coimmunoprecipitation (Co-IP) were performed in transfected BKo-myc ROS17/2.8 cells. As shown in Fig. 5g, $B K \alpha$ and integrin $\beta 1$ have almost the same localization according to fluorescent staining; the integrin $\beta 1$ protein was also detectable in the BK immunoprecipitates (Fig. 5h).

To explore the binding site between BK and integrin $\beta 1$, we co-transfected HEK293T cells with the BK-C-Flag plasmid (encoding 380-1243 amino acids, including the $\mathrm{C}$-terminal region of $\mathrm{BK} \alpha$ ) and the integrin $\beta 1$-His plasmid. Figure $5 \mathrm{i}$ shows that BK-C and integrin $\beta 1$ colocalize that was also observed in Co-IP experiments using anti-Flag antibody and anti-His antibody (Fig. 5j). These results indicate that BK (preferentially at the Cterminus of $B K \alpha$ ) binding to integrin $\beta 1$ (probably to stabilize the protein) activates Runx2 and osteoblast differentiation through FAK-ERK1/2 signaling pathway.

\section{Discussion}

In the present study, we generated a BK knockout mouse by the deletion of exon 4 of KCNMA1 (the gene encoding the pore-forming $\alpha$-subunit of $\mathrm{BK}, \mathrm{BK} \alpha)$ using the CRISPR/Cas9 strategy. By 15 weeks of age, a significant reduction in the trabecular and cortical bone of BK-deficient (BKO) female mice was revealed by $\mu \mathrm{CT}$ analysis. Importantly, defects in bone formation in these BKO mice were detected by histomorphometric analysis. The osteoblast activities, which were evaluated by osteoblast perimeter (Ob.Pm/Tb.Pm), mineral apposition rate (MAR) and bone formation rate (BFR/B.S) were decreased in BKO mice, with no or modest changes in osteoclast activity. The impaired osteoblast function was further identified by decreases in ALP activity and its ability to form mineralized nodules in BMSC cultures. This finding was further confirmed by the reduction of Runx 2 and ALP expression in the bones and BMSCs of BKO mice. These results demonstrated that the low bone mass characterized by BK deficiency was mostly, if not completely, due to a defect in bone formation from impaired osteoblast activity and osteoblast differentiation.

Osteoblasts are bone-forming cells responsible for bone matrix synthesis and its subsequent mineralization ${ }^{39}$. The cells arise from the differentiation of mesenchymal stem cells (MSCs) under the control of local (factors or signals from extracellular matrix) and systemic (e.g., PTH, $1 \alpha, 25$ $\left.[\mathrm{OH}]_{2} \mathrm{D}\right)$ factors ${ }^{40}$. Runt-related transcription factor 2 (Runx2) is the master transcription factor in this process $^{32,33,41,42}$ and could be activated via signaling 


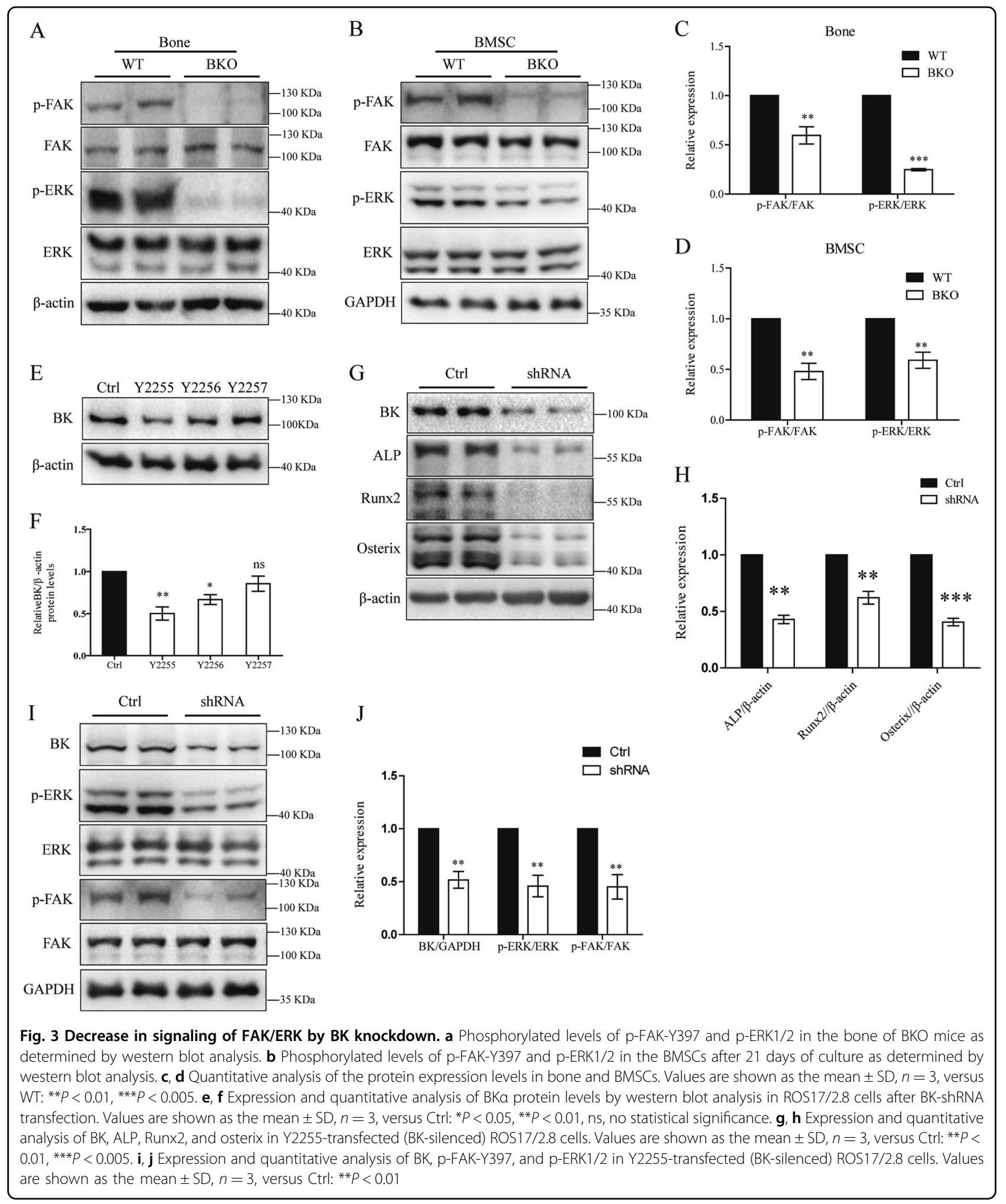

pathways, such as the mitogen-activated protein kinase (MAPK) pathway or the extracellular signal-regulated kinase (ERK) pathway ${ }^{33,34}$. The activation of Runx2 then triggers a cascade of the downstream osteoblast markers
OPN, collagen I, ALP and OCN to promote the differentiation of osteoblasts for bone matrix synthesis and mineralization $^{35}$. The decrease in the phosphorylation of focal adhesion kinase (p-FAK-Y397) and p-ERK1/2 found 

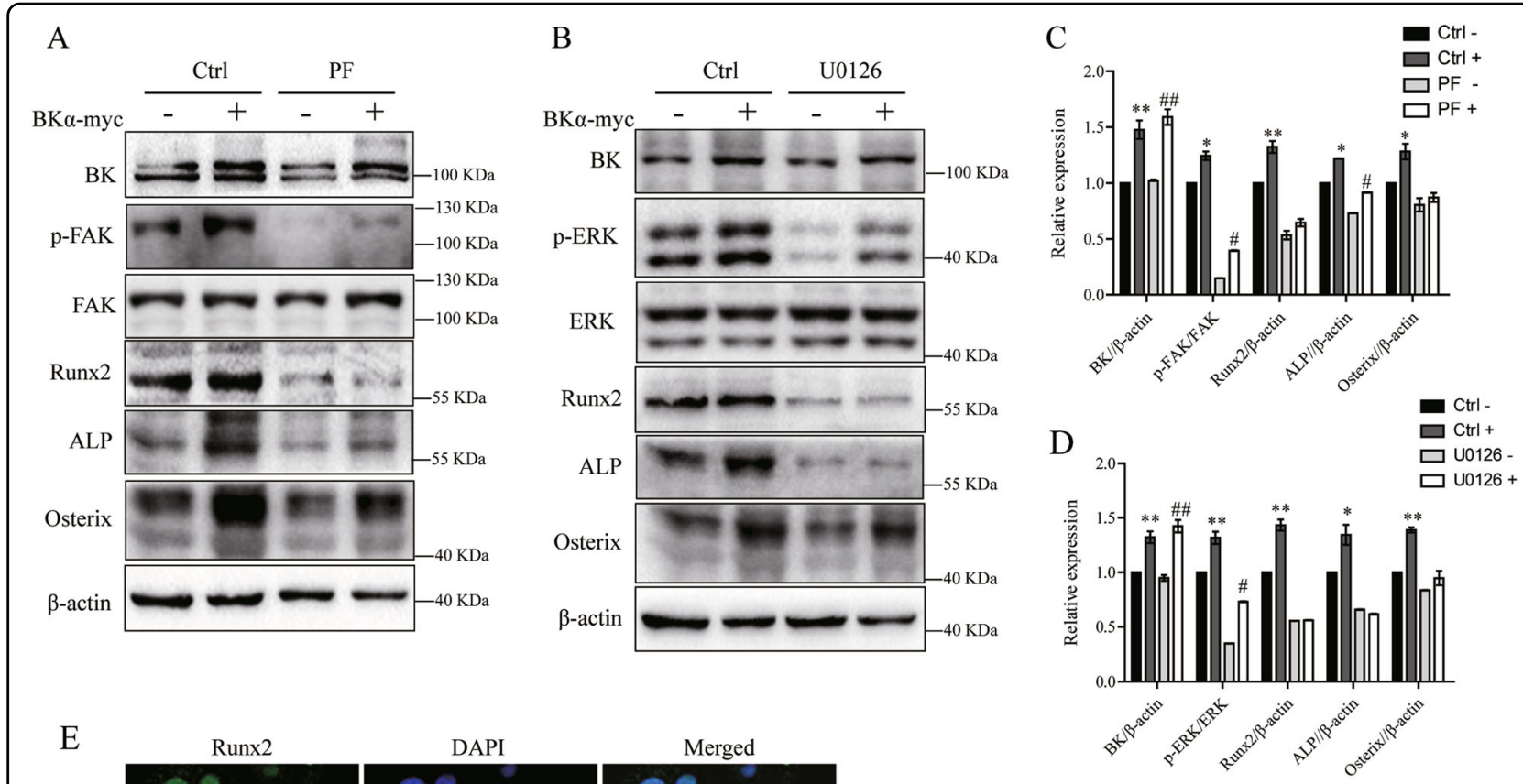

E
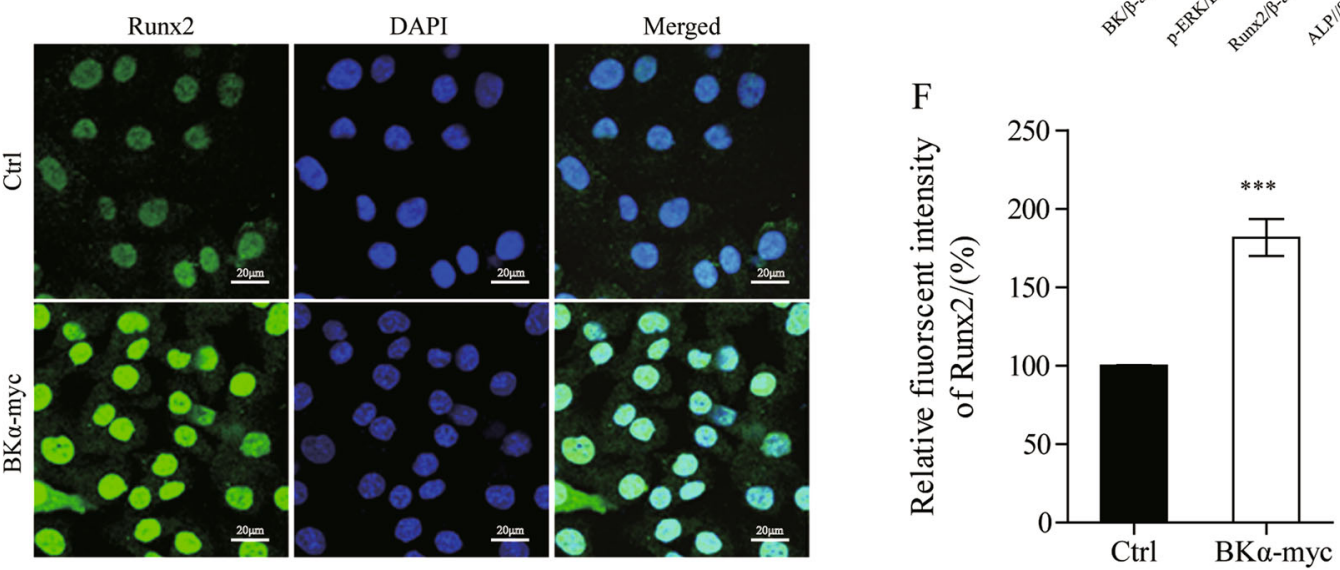

Fig. 4 Overexpression of BK increases osteogenic markers through FAK and ERK. a Western blot analysis of BK, p-FAK-Y397, RunX2, ALP, and Osterix after the BKa-myc plasmid was transfected into ROS17/2.8 cells using the FAK inhibitor PF-562271 (5 $\mu \mathrm{M})$. PF was short for PF-562271. b Western blot analysis of BK, p-ERK1/2, Runx2, ALP, and osterix in BKa-myc plasmid transfected ROS17/2.8 cells using the ERK inhibitor U0126 $(10 \mu \mathrm{M})$. c, $\mathbf{d}$ Quantitative analysis of the protein expression levels by western blot of $(\mathbf{a}, \mathbf{b})$. Values are shown as the mean $\pm \mathrm{SD}, n=3$, versus Ctrl -: ${ }^{*} P<0.05$, ${ }^{* *} P<0.01$, versus PF- or U0126 -: ${ }^{\#} P<0.05$, ${ }^{\#} P<0.01$. e Immunofluorescent staining of Runx2 in BKa-myc transfected ROS17/2.8 cells. Green is FITC-stained Runx2, and blue is DAPI-stained nuclei. $\mathbf{f}$ Quantification of Runx2 expression by immunofluorescence. Values are shown as the mean \pm $\mathrm{SD}, n=3$, versus Ctrl: ${ }^{* *} P<0.001$

in bone and BMSCs of BKO mice, as well as the reduced protein level of integrin $\beta 1$, indicated altered integrin signaling. To explore the role of integrin signaling in osteoblast defects by BK deficiency, the BK pore forming $\alpha$-subunit gene KCNMA1 were further modified in ROS17/2.8 and MC3T3-E1 osteoblasts (silenced by BK $\alpha-$ shRNA and overexpressed with the BK $\alpha$-myc plasmid), and the expression of Runx 2 and the signaling pathway were determined. In line with the findings in $\mathrm{BKO}$ mice and BKO BMSCs, silencing BK caused a decrease in Runx2 and Osterix and the downstream osteoblast markers ALP; these were paralleled with a decrease in the phosphorylation of focal adhesion kinase (p-FAK-Y397) and p-ERK1/2. Correspondently, overexpressing BK induced Runx2, osterix, and ALP, which could be abolished by inhibitors of FAK or ERK1/2. These results indicate that Runx2, acting as one of the key targets, could be activated by BK through the FAK/ERK pathway in osteoblasts.

Integrins are a family of transmembrane $\alpha / \beta$ heterodimer adhesion molecules that convey signals to (outsidein signaling) and from (inside-out signaling) the cytosol across the plasma membrane ${ }^{31,43}$. Integrins, especially integrin $\beta 1$, play important roles in bone formation. The deletion of $\beta 1$ or its regulatory factor ICAP- 1 decreases osteoblast differentiation and bone formation, leading to a 


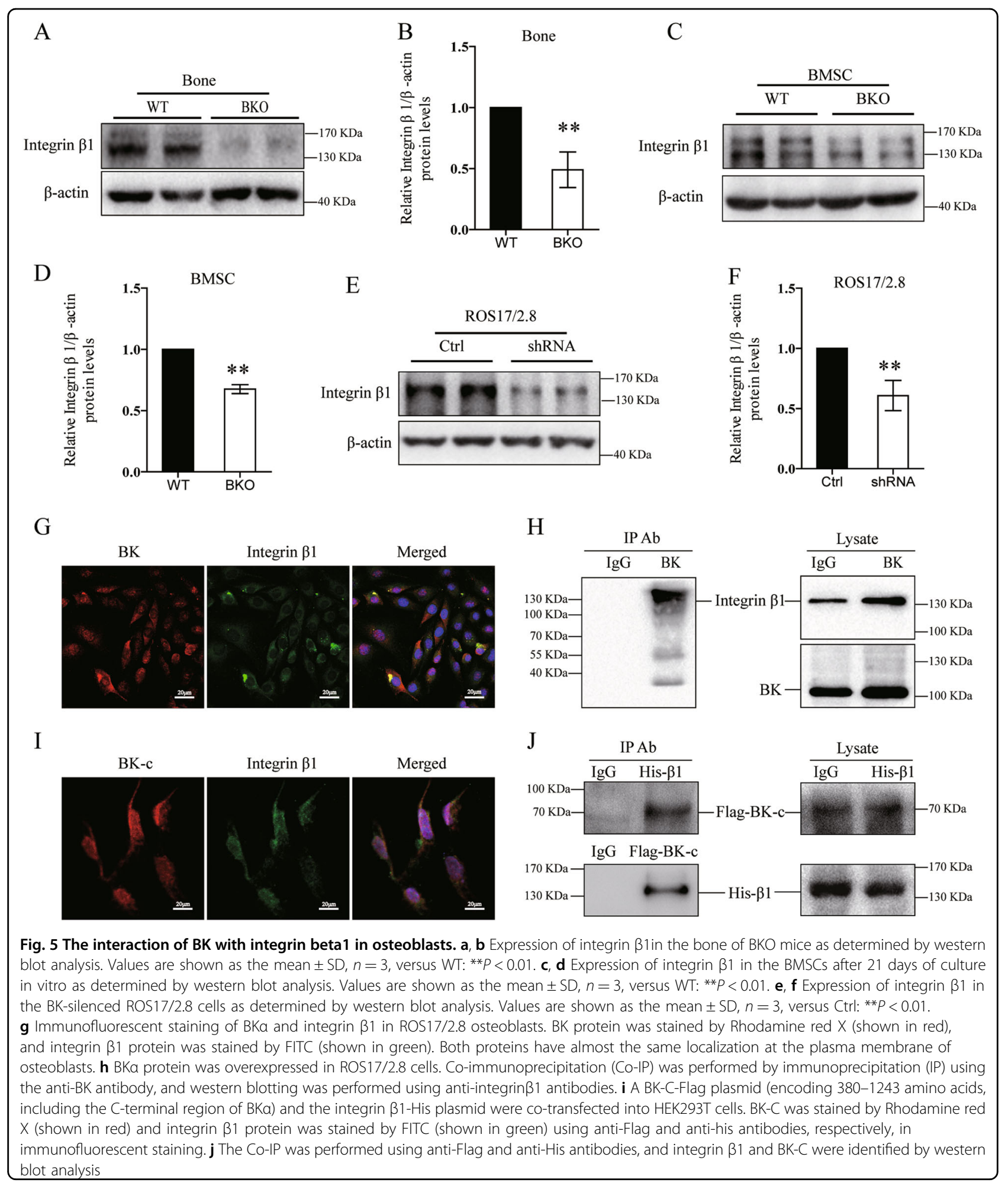

significant decrease in bone mineral density ${ }^{44,45}$. The activation of integrin $\beta 1$ leads to FAK aggregation and phosphorylation (autophosphorylation at Y397 site), thereby triggering a series of downstream signaling pathways, such as MAPK/ERK1/2, and further activating
Runx2 to promote osteoblasts differentiation ${ }^{33-35,46}$. Integrin $\beta 1$ decreased by BK deficiency in the bone and BMSCs of our BKO mice and in BK $\alpha$-silenced osteoblasts. To further explore the relationship of BK and integrin $\beta 1$, the interaction of these two molecules was evaluated in 


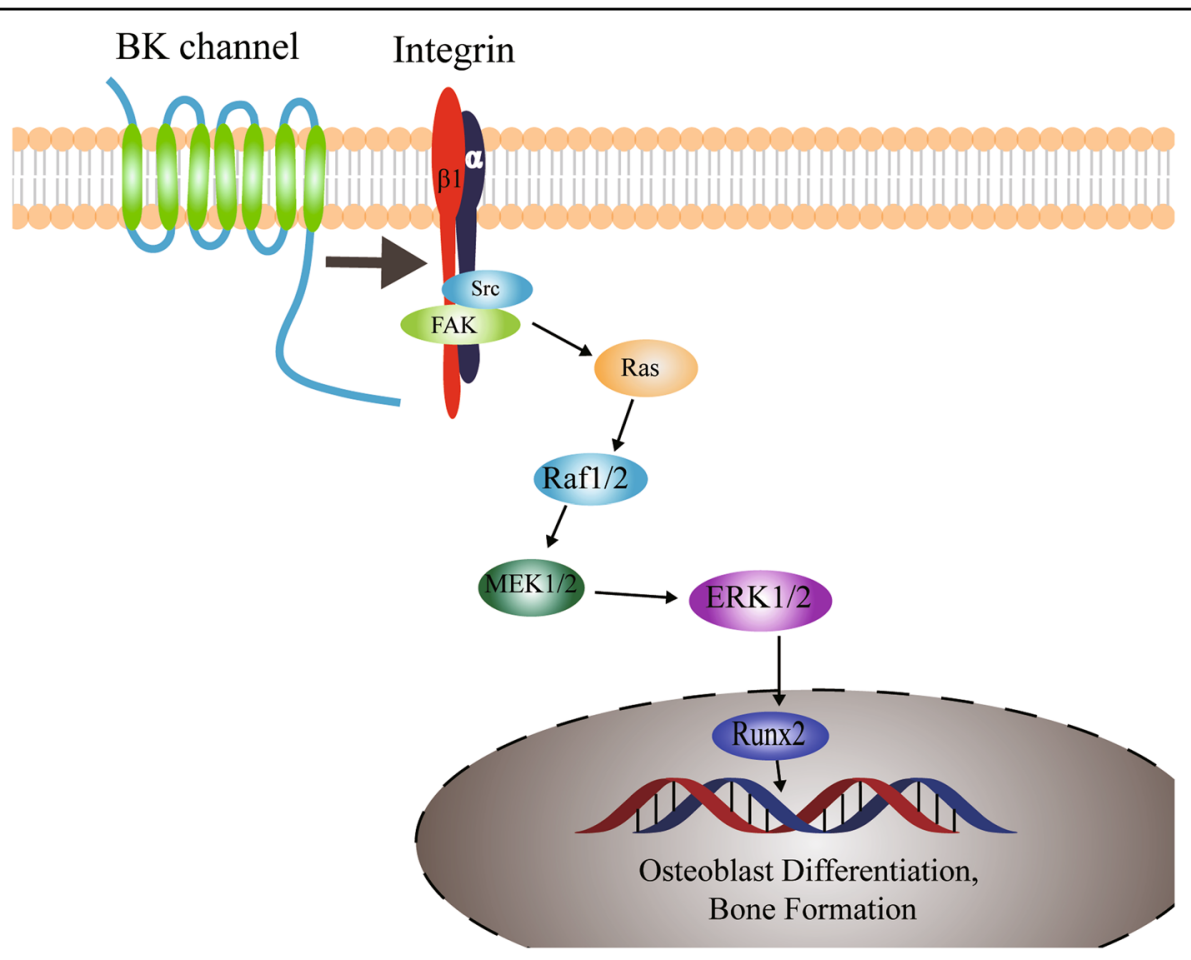

Fig. 6 The illustration of BK action in osteoblasts. BK protein (preferentially via C-terminus of BKa) binds integrin $\beta 1$ in osteoblasts. By binding to integrin $\beta 1$ (probably to stabilize the protein), BK activates the signaling of integrins, such as FAK and ERK, increases transcription factor Runx2 and accelerate osteoblast differentiation and bone formation

KCNMA1 - or integrin $\beta 1$-modified cells. As expected, the binding of full-length $\mathrm{BK} \alpha$ with integrin $\beta 1$ was confirmed by immunocytochemistry and Co-IP. Although integrin $\beta 1$ is decreased by $\mathrm{BK}$ silencing, the downregulation of integrin $\beta 1$ did not affect the expression of BK (supplemental data), indicating that BK acts upstream of integrin $\beta 1$ signaling in osteoblasts. These results suggest that integrin $\beta 1$ mediates the actions of $\mathrm{BK}$ in osteoblasts.

The BK $\alpha$-subunit consists of a transmembrane domain (including seven transmembrane segments), a short extracellular $\mathrm{N}$ terminus and a large intracellular $\mathrm{C}$-terminus. The regulator of conduction of $\mathrm{K}^{+}(\mathrm{RCK})$ in the intracellular region of the $\mathrm{C}$-terminus of $\mathrm{BK}$ is the main region for the phosphorylation of $\mathrm{BK}^{47-49}$. To determine its binding site, the C-terminus of $\mathrm{BK} \alpha$ (380-1243 amino acid, including RCK regions) and integrin $\beta 1$ were overexpressed in HEK293T cells by the co-transfection of a BK-C-Flag plasmid and integrin $\beta 1$ His plasmid. The co-expression of $\mathrm{BK}-\mathrm{C}$ and integrin $\beta 1$ was confirmed by immunocytochemistry and Co-IP experiments. These observations indicate that the C-terminus of $\mathrm{BK} \alpha$ binds to integrin $\beta 1$ (probably to stabilize the protein) and promotes osteoblast differentiation through the activation of FAK and ERK1/2.

In summary, our studies demonstrated the critical role of BK channels in maintaining skeletal integrity in
BK-deficient mice. The reduction in bone mass mostly results from defects in bone formation caused by impaired osteoblast function due to the absence of BK through integrin signaling. There is an interaction between BK protein (especially the C-terminus of $B K \alpha$ ) and integrin $\beta 1$ in osteoblasts. Interaction between BK and integrin $\beta 1$ activates integrin signaling, via FAK and ERK subsequently leading to the increase in the transcription factor Runx2 expression and accelerated osteoblast differentiation and bone formation (as illustrated in Fig. 6). Our study suggests an important role of BK in maintaining bone formation and in the pathogenesis of osteoporosis. And thus, BK channels may represent a new therapeutic target for osteoporosis.

\section{Materials and methods}

\section{Generation of BK knockout mice using the CRISPR/ Cas9 strategy}

Two pairs of oligonucleotides, (TAGGAATTTCTCT CCATTCAACCA and AAACTGGTTGAATGGAGAG CCCTT; TAGGCAGACCCTAAACCAGAACAC and AAACGTGTTCTGGTTTAGGGTCTG) for gRNA targeting on exon 4 of KCNMA1 (which encodes the poreforming $\alpha$ subunits of $\mathrm{BK})$ were transcribed in vitro. A mixture of Cas9 mRNA $(25 \mathrm{ng} / \mu \mathrm{l})$ and gRNA $(12.5 \mathrm{ng} / \mu \mathrm{l}$ per gRNA) was microinjected into the cytoplasm of 
zygotes of C57BL/6 mice (Bioray Laboratories Inc, Shanghai, China.). Several types of frame shift mutations were found in pups. One of these mutations was chosen to establish a colony (designated BKO), which carried a 532-bp fragment deletion (including the entire exon 4 of KCNMA1) from 23591392 to $23591923 \mathrm{bp}$ in the genome DNA sequence (NC 000080.6). Genotyping was performed by PCR on DNA isolated from tails using the BK primer sequences 5'-TCTCCATACTCCCTCCCTT-3' and 5'- CCTAATCCTACAAGCCTTCACT -3' (a 1531bp fragment for WT and a 1000-bp fragment for the KCNMA1 knockout gene) and confirmed by sequencing.

The $\mathrm{BKO}$ mice were established by breeding $\mathrm{BK}^{+/-}$ males and females. These breeding pairs provided ${ }^{+/+}$ (wild-type), ${ }^{+/-}$(heterozygous) and ${ }^{-/-}$(BKO) littermates and the female mice were used for a direct comparison. In all experiments, tissues were collected at the time of sacrifice for further study. All mice were maintained in a virus- and parasite-free barrier facility and exposed to a 12-h/12-h light/dark cycle. All experiments involving animals were performed according to institutionally approved and current animal care guidelines.

\section{Microcomputed tomography $(\mu \mathrm{CT})$}

Samples of tibiae and the second lumbar vertebra (L2) obtained from female mice at 15 weeks of age were dissected free of soft tissue, fixed overnight in 70\% ethanol and analyzed by $\mu \mathrm{CT}$ with a SkyScan 1172 scanner and associated analysis software (SkyScan, Belgium). Scans were performed on the whole L2 and the proximal part of the tibia. Image acquisition was performed at $59 \mathrm{kV}$ and $167 \mu \mathrm{A}$, with a $0.6^{\circ}$ rotation between frames. Thresholding $(85 / 255)$ was applied to the images to segment the bone from the background. The 3D reconstruction was performed using 2-dimensional data from scanned slices with the 3D Creator software supplied with the instrument. For lumbar reconstruction, the trabecular bone volume of interest (VOI) was drawn to include all cancellous bone in the whole cavity. For tibia reconstruction, the VOI was drawn to include 100 slices of metaphyseal spongiosa $(\sim 1 \mathrm{~mm}$ region) starting from $1 \mathrm{~mm}$ below the growth plate. The trabecular bone volume/tissue volume (BV/ TV), trabecular thickness (Tb.Th), trabecular separation (Tb.Sp) and bone mineral density (BMD) as well as the cortical bone volume (Ct.BV), cortical bone thickness (Ct. $\mathrm{Th}$ ) and cortical bone intersection surface area (Ct.Ar) were calculated as previously described ${ }^{50}$. The resolution of the $\mu \mathrm{CT}$ images was 9.92 microns.

\section{Histomorphometry}

The femur and vertebrae (L3) were removed and fixed in PLP fixative (2\% paraformaldehyde containing $0.075 \mathrm{M}$ lysine and $0.01 \mathrm{M}$ sodium periodate solution) overnight at $4^{\circ} \mathrm{C}$ and processed histologically using a modified $\operatorname{method}^{51}$. The bones were decalcified in an ethylenediamine tetra-acetic acid (EDTA) glycerol solution for 2 weeks at $4{ }^{\circ} \mathrm{C}$. Decalcified bones were dehydrated and embedded in paraffin, after which $4 \mu \mathrm{m}$ sections were cut on a rotary microtome. The sections were stained with Mayer's hematoxylin and eosin (H\&E) and histochemically tested for alkaline phosphatase (ALP) activity using a BCIP/NBT kit (C3206, Beyotime Biotechnology, China) and for tartrate-resistant acid phosphatase (TRAP) activity using a TRACP kit (387-A Leukocyte Acid Phosphatase Kit, Sigma-Aldrich, USA).

After staining, images of fields in the metaphyseal area below the growth plate were viewed and imaged with a microscope (Imager.M2, Zeiss, Germany). Images of micrographs from single sections were digitally recorded using a rectangular template, and the recordings were processed for histomorphometric measurements and analyzed using Image-Pro Plus software (Media Cybernetics, USA). The parameters obtained for bone formation were the ALP-positive osteoblast perimeter per trabecular perimeter (Ob.Pm/Tb.Pm, \%). The parameter measured for bone resorption was the TRAP-positive osteoclast perimeter per trabecular perimeter (Oc.Pm/Tb.Pm, \%).

\section{Tetracycline and calcein labeling}

Tetracycline $(30 \mathrm{mg} / \mathrm{kg})$ and calcein $(10 \mathrm{mg} / \mathrm{kg})(\mathrm{C}-0875$, Sigma, USA) were administered by intraperitoneal injection 10 and 3 days prior to sacrifice. The lumbar vertebrae were harvested and fixed overnight in 70\% ethanol. The samples were dehydrated for 1 day in $30 \%$ sucrose, embedded in OCT (4583, Tissue-Tek, Jap) and then frozen in the vapor phase of liquid nitrogen. Serial sections were cut on a freeze microtome, and the freshly cut surface of each section was viewed and imaged using fluorescence microscopy (E600, Nikon, Jap) with a Digital Camera (DXM1200, Nikon) and attached software. The single labeled perimeter (sL.Pm), double labeled perimeter (dL.Pm), interlabel width (IrL. $\mathrm{Wi})$, and Trabecular perimeter (Tb.Pm) were measured using Image Pro Plus software. The mineral apposition rate (MAR; MAR $=$ interlabel width $/ 7, \mu \mathrm{m}$ per day) and bone formation rate (BFR/BS; BFR/BS $=($ sL.Pm $/ 2+\mathrm{dL} . \mathrm{Pm}) /$ Tb.Pm*MAR) were calculated.

\section{Cell cultures}

bone marrow mesenchyma stem cells (BMSCs) were flushed out from the femur and tibia of mice ( $>2$ months old) with DMEM (Gibco, USA) containing $100 \mathrm{IU} / \mathrm{ml}$ penicillin, $100 \mathrm{mg} / \mathrm{ml}$ streptomycin (Gibco) and 10\% fetal bovine serum (FBS, Gibco) and supplemented with $50 \mu \mathrm{g} / \mathrm{ml}$ ascorbic acid, $10 \mathrm{mM} ß$-glycerophosphate, and $10^{-8} \mathrm{~mol} / \mathrm{L}$ dexamethasone (differentiation medium). The cells were dispersed by repeated pipetting, and a singlecell suspension was achieved by forcefully expelling the cells through a 22-gauge syringe needle. The cells 
from two mice were pooled and cultured in one 6- or 24well plate (Nunc, USA) in differentiation medium. The cultures were maintained for 14 days for ALP staining or 21 days for Alizarin Red staining and western blot analysis. At the end of the culture period, the cells were washed with PBS, fixed with PLP fixative, and stained for ALP using the BCIP/NBT kit (Beyotime Biotechnology, Jiangsu, China) by a standard procedure or stained in $1 \mathrm{mg} / \mathrm{ml}$ Alizarin Red solution (Yeasen, Shanghai, China) for $30 \mathrm{~min}$ at room temperature as previously described. After washing with distilled water and drying in air, images of the stained plates were taken.

ROS17/2.8 cells, a rat osteoblastic cell line, were a kind gift from Dr. Sandra Guggino (Johns Hopkins University, Baltimore, MD). ROS17/2.8 cells were cultured in Ham's F-12 nutrient mixture medium (Sigma-Aldrich, USA) containing $\mathrm{NaHCO} 3$ and supplemented with $10 \%$ FBS, $100 \mathrm{U} / \mathrm{ml}$ penicillin and $100 \mathrm{mg} / \mathrm{ml}$ streptomycin. HEK293T cells were cultured in Dulbecco's Modified Eagle Medium (Gibco, USA) supplemented with 10\% FBS, $100 \mathrm{U} / \mathrm{ml}$ penicillin and $100 \mathrm{mg} / \mathrm{ml}$ streptomycin. The cells were cultured in a humidified atmosphere of $95 \%$ air and $5 \% \mathrm{CO}_{2}$ at $37^{\circ} \mathrm{C}$ and subcultured every $2-3$ days. For the experiments, the cells were trypsinized at approximately $90 \%$ confluence and seeded onto 6 -well plates at a density of $2.5 \times 10^{4} / \mathrm{ml}$.

\section{RNA interference}

A series of small hairpin RNA (shRNA) targeted to mRNA of BK $\alpha$ (KCNMA1; NM-031828) or integrin $\beta 1$ (ITGB1; NM-017022) were designed and constructed in the lentivirus vector Plko-CMV-G\&PR-U6-shRNA (Obio Technology (Shanghai) Co. Ltd, China) according to the manufacturer's protocol. Three shRNA plasmids targeted to KCNMA1 (Y2255, targeted to GCGGTTTATTGCA GCCAATGA, Y2256 to GCTTAAGCTCCTGATGATA GC, and Y2257 to GCATCTTGGCGTCACTCAACA) and two shRNA plasmids targeted to ITGB1 (Y2652 to CCACAACAGCTGCTTCTAA and Y2653 to GGAGGA TTACTTCAGACTT) were obtained. The plasmid Y007 targeted to TTCTCCGAACGTGTCACGT was used as a negative control. The shRNA plasmids were transfected into ROS 17/2.8 cells in 6-well plates using Lipofectamine 3000 (L3000-015, Invitrogen, USA) according to the manufacturer's protocol. Four $\mu \mathrm{g} / \mathrm{ml}$ puromycin was added for selection on the second day of transfection. Two days later, the cell proteins were extracted for analysis by western blotting.

\section{BK overexpression}

The BKa-myc plasmid was transfected into ROS 17/2.8 cells in 6-well plates using Lipofectamine 3000. The medium was refreshed $24 \mathrm{~h}$ later, and $10 \mu \mathrm{M}$ ERK1/2 inhibitor U0126 (S1102, Selleck, USA) or $5 \mu \mathrm{M}$ FAK inhibitor PF-562271 (S2890, Selleck) was added as needed. The cells were collected for protein analysis 2 days later. The BK-C-Flag plasmid (code for 380-1243 amino acids, including the region of the c-terminus of $B K \alpha$ ) and the integrin $\beta 1$-His plasmid were designed by Vigene Biosciences (Rockville, MD, USA). The BK $\alpha$-myc plasmid or BK-C-Flag plasmid with integrin $\beta 1$-His were cotransfected into HEK293T cells in 6-well plates using Lipofectamine 3000 . The cells were collected for protein analysis 2 days later.

\section{Immunofluorescent staining}

Immunocytochemistry analysis was performed according to a previous report ${ }^{21}$. The cells were seeded onto slides. At $70 \%$ confluence, the cells were fixed in $4 \%$ paraformaldehyde in PBS buffer for $15 \mathrm{~min}$. Following washes with PBS, the cells were permeabilized in freshly prepared $0.1 \%$ Triton X-100 for $10 \mathrm{~min}$ and blocked in $1 \%$ bovine serum albumin (BSA) in PBS buffer for $1 \mathrm{~h}$ at room temperature. The cells were incubated with primary antibodies directed against either runx2 (1:200, ab76956, Abcam), the $\alpha$-subunit of BK (1:100, ab99046, Abcam, USA), integrin beta1 (1:100, 1798-1, Epitomics, USA), Flag (1:200, Rabbit Monoclonal Anti-Flag antibody, F2555, Sigma) or His (1:200, SAB1306084, Sigma) at $4{ }^{\circ} \mathrm{C}$ overnight. The secondary Alexa Fluor 647conjugated anti-mouse antibody (Jackson Immuno Research, USA) was used at a 1:200 dilution for $2 \mathrm{~h}$ at room temperature protected from light. Following washes with PBS, the cells were stained with DAPI for $5 \mathrm{~min}$. The slides were mounted with SlowFade ${ }^{\mathrm{TM}}$ Gold Antifade Mountant (S36936, Invitrogen), and images were captured using a Leica TCS SP5 confocal microscope (Leica, Germany) at room temperature with $\times 10$ and $\times 40$ objective lenses.

\section{Quantitative real-time PCR}

Total RNA was extracted from long bones using Trizol reagent (15596, Invitrogen) according to the manufacturer's protocol. The total RNA was reversetranscribed to cDNA using the QuantiTect Rev Transcription Kits (205311, Qiagen, USA). The number of cDNA molecules in the reverse-transcribed samples was determined by real-time PCR analysis using a modified method with QuantiTect SYBR Green PCR Kits (204143, Qiagen) on an Mx3000P Real-Time PCR system (Stratagene, USA) ${ }^{52}$. Primers with the following sequences were obtained from BGI (BGI, China): RUNX2, 5'-TTCAA CGATCTGAGATTTGTG G-3' and 5'- GGATGAGGA ATGCGCCCTA-3'; ALP, 5'-CCAACTCTTTTGTGCCA GAGA-3' and 5'-GGCTACATTGGTGTTGAGC TTT T-3'; RANKL, 5'-CAGCATCGCTCTGTTCCTGTA-3' and 5'-CTGCGTTTTC ATGGAGTCTCA-3'; OPG, 5'-ACCCAGAAACTGGTCATCAGC-3' and 5'-CTGC 
AATACACACACTCATCACT-3'; and GAPDH, 5'-AGG TCGGTGTGAACGGATTT G-3' and 5'-TGTAGACC ATGTAGTTGAGGTCA-3'. The PCR reactions included $12.5 \mu \mathrm{l}$ of SYBR Green I master mix, $0.25 \mu \mathrm{M}$ of each 5' and 3' primer, $2 \mu \mathrm{l}$ of sample cDNA and $\mathrm{H}_{2} \mathrm{O}$ to a final volume of $25 \mu \mathrm{l}$. A melting curve was obtained at the end of each run to discriminate specific from nonspecific cDNA products. The cDNA content was normalized by subtracting the cycle numbers of GAPDH from those of the target gene $(\Delta \mathrm{Ct}=\mathrm{Ct}$ of target gene-Ct of GAPDH), and gene expression levels were calculated using the $2^{-}$ $(\Delta \mathrm{Ct})$ method.

\section{Western blotting analysis}

Total proteins from the long bones or cells were analyzed by western blotting using a modified method $^{21}$. Briefly, the bone powder and cells were lysed with RIPA buffer supplemented with $1 \%$ protease inhibitor cocktail (P0013B; Beyotime) for $30 \mathrm{~min}$ on ice. Insoluble materials were removed by centrifuging at $12,000 \times g$ for $10 \mathrm{~min}$, and the supernatants were collected. The protein amount was then quantified with the BCA protein assay (P0012; Beyotime) using BSA as a standard. The sample protein was denatured in boiling water for $5 \mathrm{~min}$ in SDS-PAGE sample loading buffer (P0015, Beyotime). Aliquots of the samples $(40 \mu \mathrm{g})$ were then subjected to SDS-PAGE on $12 \%$ gels under reducing conditions and electroblotted onto PVDF membranes (Ipvh00010; Millipore, USA). The membranes were blocked with $5 \%$ fat-free dry milk in TBST $(0.1 \%$ Tween-20 and $0.1 \mathrm{M} \mathrm{NaCl}$ in $0.1 \mathrm{M}$ Tris- $\mathrm{HCl}, \mathrm{pH} 7.5$ ) for $2 \mathrm{~h}$ at room temperature and then incubated with primary antibodies at $4{ }^{\circ} \mathrm{C}$ overnight. The antibodies include anti-BK (APC-107, Alomone Labs, Israel), antiRunx2 (ab76956, Abcam, USA), anti-integrin $\beta 1$ (17981, Epitomics), anti-FAK (12636-1-AP, Proteintech, USA), anti-FAK-Y397 (EP2016Y, Abcam), anti-Phospho-p44/42 MAPK (ERK1/2) (4730, Cell Signaling Technology, USA), anti-p44/42 MAPK (ERK1/2) (9102, Cell Signaling Technology), anti-Osterix (ab209484, Abcam), anti-ALP (AF1030, Beytime), anti-Flag (1:200, Rabbit Monoclonal Anti-Flag antibody, F2555, Sigma), anti-myc (Santa Cruz Biotechnology, USA), anti-his (2365, Cell Signaling Technology), anti- $\beta$-actin (ab8226, Abcam), and anti-GAPDH (ab9485, Abcam). The membranes were then incubated with horseradish peroxidase-conjugated secondary antibody (1:5000; Santa Cruz Biotechnology) at room temperature for $1 \mathrm{~h}$, followed by chemiluminescence detection (P0018, Beyotime). Each incubation step was followed by three washes $(10 \mathrm{~min}$ each) with TBST. The protein bands were quantitatively analyzed by using an image analysis system (Quantity One software; BioRad ChemiDoc, BioRad, USA).

\section{Co-Immunoprecipitation (Co-IP)}

The interaction of BK with integrins was analyzed by Co-IP. The cells were lysed with RIPA buffer as described above. One portion of the supernatant was denatured in boiling water in SDS-PAGE sample loading buffer for whole cell lysates (input). The other portion of the supernatant $(\sim 1 \mathrm{mg}$ protein in $500-800 \mu \mathrm{l})$ was incubated with an IP antibody (10 $\mu \mathrm{l}, \sim 2-5 \mu \mathrm{g}$ ) (anti-BK, anti-myc antibody, anti-Flag antibody or anti-His antibody) for $1 \mathrm{~h}$ at $4{ }^{\circ} \mathrm{C}$ in a rolling incubator. Protein $\mathrm{A} / \mathrm{G}$ agarose beads (20-40 $\mu$ l, sc-2003, Santa Cruz Biotechnology) were then added and incubated overnight at $4{ }^{\circ} \mathrm{C}$ in a rolling incubator. The immunoprecipitates (beads) were collected by centrifugation at $1000 \times g$ for $5 \mathrm{~min}$ at $4^{\circ} \mathrm{C}$. The pelleted beads were washed three times with pre-cold RIPA buffer $\left(1000 \times g\right.$ for $5 \mathrm{~min}$ at $\left.4{ }^{\circ} \mathrm{C}\right)$ and eluted in boiling water for $5 \mathrm{~min}$ in $30-50 \mu \mathrm{l} 2 \times$ loading buffer. The bound proteins were detected by western blot analysis.

\section{Statistical analysis}

Data are presented as means \pm SD and repeated at least in three independent experiments. Statistical comparisons were carried out using one-way ANOVA ( $>2$ groups) or unpaired two-tail Student's $t$-test (two group), and $P$-value $<0.05$ was considered significant.

\section{Acknowledgements}

This work was supported by grants from the National Natural Science Foundation of China (No. 81773801 and No. 81573478 to X. Zhang, No. 81373442 to K. Yu and No. 81603410 to J. Tao), NBR 973 Program of China (No. 2013 CB932500 to K. Yu), NST Major Project of China (No. 2018ZX09711002-008 to K. Yu), and Shanghai Science and Technology Innovation (No. 14140903202 to $X$. Zhang).

\section{Author details}

'Department of Pharmacology, School of Pharmacy, Fudan University, Shanghai 201203, China. ${ }^{2}$ Department of Bone Metabolism, Institute of Radiation Medicine, Fudan University, Shanghai 200032, China. ${ }^{3}$ Department of Nephrology, Putuo Hospital, Shanghai University of Traditional Chinese Medicine, Shanghai 200062, China. ${ }^{4}$ Department of Medicine, Renal Division, Emory University School of Medicine, Atlanta, GA 30322, USA. ${ }^{5}$ Section of Nephrology, Atlanta Veteran Administration Medical Center, Decatur, GA 30033, USA. ${ }^{6}$ Division of Cardiovascular Medicine, Radcliffe Department of Medicine, University of Oxford, John Radcliffe Hospital, Oxford OX3 9DU, UK

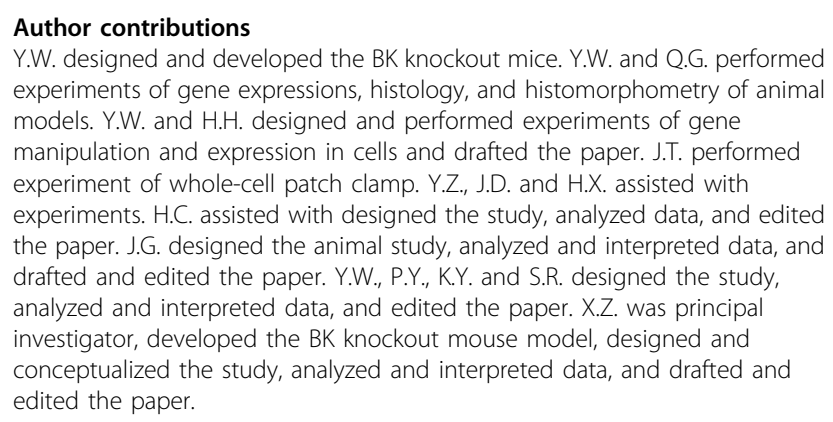
experiments of gene expressions, histology, and histomorphometry of animal models. Y.W. and H.H. designed and performed experiments of gene manipulation and expression in cells and drafted the paper. J.T. performed experiment of whole-cell patch clamp. Y.Z., J.D. and H.X. assisted with experiments. H.C. assisted with designed the study, analyzed data, and edited the paper. J.G. designed the animal study, analyzed and interpreted data, and drafted and edited the paper. Y.W., P.Y., K.Y. and S.R. designed the study, analyzed and interpreted data, and edited the paper. X.Z. was principal investigator, developed the BK knockout mouse model, designed and conceptualized the study, analyzed and interpreted data, and drafted and edited the paper.

Conflict of interest

The authors declare that they have no conflict of interest. 


\section{Publisher's note}

Springer Nature remains neutral with regard to jurisdictional claims in

published maps and institutional affiliations.

Supplementary Information accompanies this paper at (https://doi.org/ 10.1038/s41419-019-1972-8).

Received: 4 June 2019 Revised: 4 September 2019 Accepted: 5 September 2019

Published online: 30 September 2019

\section{References}

1. $L G, R$. Overview of Pathogenesis. in Primer on the metabolic bone disease and disorders of mineral metabolism (ed. Rosen CJ) Chapter 39, 203-206 (2008).

2. Watts, N. B. \& Manson, J. E. Osteoporosis and fracture risk evaluation and management: shared decision making in clinical practice. JAMA 317, 253-254 (2017).

3. Kiel, D. P., Rosen, C. J. \& Dempster, A. D. Chapter 20. Age-Related Bone Loss. American Society for Bone and Mineral Research, 98-102 (2008).

4. Marie, P. J. Bone cell senescence: mechanisms and perspectives. J. Bone Miner. Res. 29, 1311-1321 (2014)

5. Manolagas, S. C. From estrogen-centric to aging and oxidative stress: a revised perspective of the pathogenesis of osteoporosis. Endocr. Rev. 31, 266-300 (2010).

6. Sausbier, U. et al. Osteopenia due to enhanced cathepsin $\mathrm{K}$ release by BK channel ablation in osteoclasts. PloS one 6, e21168 (2011).

7. Mackinnon, R. Potassium channels. FEBS Lett. 555, 62-65 (2003).

8. Tian, C. et al. Potassium channels: structures, diseases, and modulators. Chem. Biol. Drug Des. 83, 1-26 (2014).

9. Kuang, Q., Purhonen, P. \& Hebert, H. Structure of potassium channels. Cell. Mol. Life Sci. 72, 3677-3693 (2015).

10. Latorre, R. et al. Molecular determinants of BK Channel functional diversity and functioning. Physiological Rev. 97, 39-87 (2017).

11. Rothberg, B. S. The BK channel: a vital link between cellular calcium and electrical signaling. Protein cell 3, 883-892 (2012).

12. Morera, F. J. et al. Voltage-dependent BK and Hv1 channels expressed in nonexcitable tissues: new therapeutics opportunities as targets in human diseases. Pharmacol. Res. 101, 56-64 (2015).

13. Ge, L. et al. Big Potassium (BK) ion channels in biology, disease and possible targets for cancer immunotherapy. Int. Immunopharmacol. 22, 427-443 (2014).

14. Hirukawa, K., Muraki, K., Ohya, S., Imaizumi, Y. \& Togari, A. Electrophysiological properties of a novel $\mathrm{Ca}(2+)$-activated $\mathrm{K}(+)$ channel expressed in human osteoblasts. Calcif. Tissue Int. 83, 222-229 (2008).

15. Ravesloot, J. H., van Houten, R. J., Ypey, D. L. \& Nijweide, P. J. Identification of Ca $(2+)$-activated $\mathrm{K}+$ channels in cells of embryonic chick osteoblast cultures. J. Bone Miner. Res. 5, 1201-1210 (1990).

16. Henney, N. C. et al. A large-conductance (BK) potassium channel subtype affects both growth and mineralization of human osteoblasts. Am. J. Physiol. Cell Physiol. 297, C1397-C1408 (2009).

17. Moreau, R., Aubin, R., Lapointe, J. Y. \& Lajeunesse, D. Pharmacological and biochemical evidence for the regulation of osteocalcin secretion by potassium channels in human osteoblast-like MG-63 cells. J. Bone Miner. Res. 12, 1984-1992 (1997).

18. Moreau, R., Hurst, A. M., Lapointe, J. Y. \& Lajeunesse, D. Activation of maxi-K channels by parathyroid hormone and prostaglandin E2 in human osteoblast bone cells. J. Membr. Biol. 150, 175-184 (1996).

19. Weskamp, M., Seidl, W. \& Grissmer, S. Characterization of the increase in [Ca(2 $+)$ ](i) during hypotonic shock and the involvement of $\mathrm{Ca}(2+)$-activated $\mathrm{K}(+)$ channels in the regulatory volume decrease in human osteoblast-like cells. J. Membr. Biol. 178, 11-20 (2000).

20. Dixon, S. J., Aubin, J. E. \& Dainty, J. Electrophysiology of a clonal osteoblast-like cell line: evidence for the existence of a Ca2+-activated $\mathrm{K}+$ conductance. J. Membr. Biol. 80, 49-58 (1984).

21. Hei, H. et al. BK Knockout by TALEN-mediated gene targeting in osteoblasts: KCNMA1 determines the proliferation and differentiation of osteoblasts. Mol. Cells 39, 530-535 (2016).

22. Heubach, J. F. et al. Electrophysiological properties of human mesenchymal stem cells. J. Physiol. 554, 659-672 (2004).
23. Li, G. R., Sun, H., Deng, X. \& Lau, C. P. Characterization of ionic currents in human mesenchymal stem cells from bone marrow. Stem Cells (Dayt., Ohio) 23, 371-382 (2005).

24. Zhang, Y. Y. et al. BKCa and hEag1 channels regulate cell proliferation and differentiation in human bone marrow-derived mesenchymal stem cells. J. Cell. Physiol. 229, 202-212 (2014).

25. Davidson, R. M. Membrane stretch activates a high-conductance $\mathrm{K}+$ channel in G292 osteoblastic-like cells. J. Membr. Biol. 131, 81-92 (1993).

26. Allard, B., Couble, M. L., Magloire, H. \& Bleicher, F. Characterization and gene expression of high conductance calcium-activated potassium channels displaying mechanosensitivity in human odontoblasts. J. Biol. Chem. 275, 25556-25561 (2000).

27. Rezzonico, R. et al. Focal adhesion kinase pp125FAK interacts with the large conductance calcium-activated hSlo potassium channel in human osteoblasts: potential role in mechanotransduction. J. Bone Miner. Res. 18, 1863-1871 (2003).

28. Rajshankar, D., Wang, Y. \& McCulloch, C. A. Osteogenesis requires FAKdependent collagen synthesis by fibroblasts and osteoblasts. FASEB J. 31, 937-953 (2017).

29. $\mathrm{Hu}$, J. et al. Focal adhesion kinase signaling mediated the enhancement of osteogenesis of human mesenchymal stem cells induced by extracorporeal shockwave. Sci. Rep. 6, 20875 (2016).

30. Sun, C. et al. FAK promotes osteoblast progenitor cell proliferation and differentiation by enhancing wnt signaling. J. Bone Miner. Res. 31, 2227-2238 (2016).

31. Pommerenke, $\mathrm{H}$. et al. The mode of mechanical integrin stressing controls intracellular signaling in osteoblasts. J. Bone Miner. Res. 17, 603-611 (2002).

32. Komori, T. Signaling networks in RUNX2-dependent bone development. J. Cell. Biochem. 112, 750-755 (2011)

33. Li, Y., Ge, C. \& Franceschi, R. T. MAP kinase-dependent RUNX2 phosphorylation is necessary for epigenetic modification of chromatin during osteoblast differentiation. J. Cell. Physiol. 232, 2427-2435 (2017).

34. Ge, C. et al. Interactions between extracellular signal-regulated kinase 1/2 and p38 MAP kinase pathways in the control of RUNX2 phosphorylation and transcriptional activity. J. Bone Miner. Res. 27, 538-551 (2012).

35. Ge, C. et al. Reciprocal control of osteogenic and adipogenic differentiation by ERK/MAP kinase phosphorylation of Runx2 and PPARgamma Transcription factors. J. Cell. Physiol. 231, 587-596 (2016).

36. Rezzonico, R. et al. Prostaglandin E2 induces interaction between hSlo potassium channel and Syk tyrosine kinase in osteosarcoma cells. J. Bone Miner. Res. 17, 869-878 (2002).

37. Hristov, K. L., Parajuli, S. P., Provence, A., Rovner, E. S. \& Petkov, G. V. Nongenomic modulation of the large conductance voltage- and $\mathrm{Ca}(2+)$-activated $\mathrm{K}(+)$ channels by estrogen: A novel regulatory mechanism in human detrusor smooth muscle. Physiol. Rep. 5, https:// doi.org/10.14814/phy2.13351 (2017).

38. Danesh, S. M., Kundu, P., Lu, R., Stefani, E. \& Toro, L. Distinct transcriptional regulation of human large conductance voltage- and calcium-activated $\mathrm{K}+$ channel gene (hSlo1) by activated estrogen receptor alpha and c-Src tyrosine kinase. J. Biol. Chem. 286, 31064-31071 (2011).

39. Siddiqui, J. A. \& Partridge, N. C. Physiological bone remodeling: systemic regulation and growth factor involvement. Physiology (Bethesda). 31, 233-245 (2016).

40. Long, F. Building strong bones: molecular regulation of the osteoblast lineage. Nat. Rev. Mol. cell Biol. 13, 27-38 (2011).

41. Lian, J. B. \& Stein, G. S. Runx2/Cbfa1: a multifunctional regulator of bone formation. Curr. Pharm. Des. 9, 2677-2685 (2003).

42. Jun, J. H. et al. BMP2-activated Erk/MAP kinase stabilizes Runx2 by increasing p300 levels and histone acetyltransferase activity. J. Biol. Chem. 285, 36410-36419 (2010).

43. Marie, P. J., Hay, E. \& Saidak, Z. Integrin and cadherin signaling in bone: role and potential therapeutic targets. Trends Endocrinol. Metab. 25, 567-575 (2014).

44. Brunner, M. et al. Osteoblast mineralization requires beta1 integrin/ICAP-1dependent fibronectin deposition. J. cell Biol. 194, 307-322 (2011).

45. Globus, R. K. et al. Skeletal phenotype of growing transgenic mice that express a function-perturbing form of beta1 integrin in osteoblasts. Calcif. Tissue Int. 76, 39-49 (2005).

46. Kim, S. H., Turnbull, J. \& Guimond, S. Extracellular matrix and cell signalling: the dynamic cooperation of integrin, proteoglycan and growth factor receptor. J. Endocrinol. 209, 139-151 (2011). 
47. N'Gouemo, P. Targeting BK (big potassium) channels in epilepsy. Expert Opin. Therapeutic Targets 15, 1283-1295 (2011).

48. Giraldez, T. \& Rothberg, B. S. Understanding the conformational motions of RCK gating rings. J. Gen. Physiol. 149, 431-441 (2017)

49. Savalli, N., Pantazis, A., Yusifov, T., Sigg, D. \& Olcese, R. The contribution of RCK domains to human BK channel allosteric activation. J. Biol. Chem. 287, 21741-21750 (2012)
50. Bouxsein, M. L. et al. Guidelines for assessment of bone microstructure in rodents using micro-computed tomography. J. Bone Miner. Res. 25, 1468-1486 (2010).

51. Gao, J. et al. Altered ovarian function affects skeletal homeostasis independent of the action of follicle-stimulating hormone. Endocrinology 148, 2613-2621 (2007).

52. Tang, W. J. et al. Autocrine/paracrine action of vitamin D on FGF23 expression in cultured rat osteoblasts. Calcif. Tissue Int. 86, 404-410 (2010). 